MSUHEP-940715

July 15, 1994

\title{
Multiplicity Distributions and Rapidity Gaps
}

\author{
Jon Pumplin \\ Physics and Astronomy Department \\ Michigan State University \\ East Lansing MI 48824, U.S.A.
}

\begin{abstract}
We examine the phenomenology of particle multiplicity distributions, with special emphasis on the low multiplicities that are a background to the study of rapidity gaps. In particular, we analyze the multiplicity distribution in a rapidity interval between two jets, using the HERWIG QCD simulation with some necessary modifications. The distribution is not of the "negative binomial" form, and displays an anomalous enhancement at zero multiplicity. Some useful mathematical tools for working with multiplicity distributions are presented. It is demonstrated that ignoring particles with $p_{\perp}<0.2 \mathrm{GeV} / \mathrm{c}$ has theoretical advantages, in addition to being convenient experimentally.
\end{abstract}




\section{Introduction}

The probabilities for various numbers of hadrons to be produced in a high energy collision, in a fixed region of phase space that is usually defined by a range of pseudo-rapidity, is known as the multiplicity distribution. Attempts have been made to understand multiplicity distributions on the basis of intuitive notions of branching and decay of "clusters" [1, 2]. Approaches with an explicit basis in QCD have also been made for regimes where an underlying hard scattering permits perturbative techniques [3]. QCD simulation programs such as HERWIG [4] include elements of both of these approaches.

Previous work on the multiplicity distribution $\left\{P_{n}\right\}$ has centered on KNO scaling and its violation [5], "intermittency" [6], and the factorial moments $\langle n\rangle,\langle n(n-1)\rangle, \ldots$ [7]. These related concepts emphasize average and largerthan-average multiplicities, which reflect the multiple soft jet production that is characteristic of QCD at high energy. In this paper, we instead focus on the region of low multiplicity.

At the extreme low end of the multiplicity distribution, one encounters the physics of rapidity gaps, which can be defined as regions of length $\Delta y \gtrsim 3$ in rapidity that contain no final-state particles. Rapidity gaps offer a unique insight into the workings of QCD. They can in principle be made by the exchange of a color-singlet object, such as an appropriate state of two or more gluons. They can also be considered - by definition - to be a facet of the mysterious pomeron that also governs elastic and diffractive scattering.

A particularly interesting type of rapidity gap occurs when the gap lies between two high- $p_{\perp}$ jets that are widely separated in rapidity and approximately back-to-back in azimuthal angle [8, 9, 10]. In this paper, we study the multiplicity distribution in a region between two such jets, resulting from non-pomeron physics; since that is an unavoidable background to probing rapidity gap physics.

The major detectors CDF and D $\varnothing$ at the Tevatron $(\bar{p} p$ at $\sqrt{s}=1800 \mathrm{GeV})$, and Zeus and H1 at HERA [1]] $\left(e^{-} p\right.$ at $\left.\sqrt{s}=300 \mathrm{GeV}\right)$, can be used to study rapidity gaps experimentally. However, the range in pseudo-rapidity where these detectors are most sensitive, reduced to leave room for jet evidence of hard scattering, is not very large. I It is therefore important to estimate the

\footnotetext{
${ }^{1}$ The coverage in $\eta$ could in principle be extended using scintillation counters as "gap detectors". A detector upgrade of this type should be relatively simple, since it is not necessary to have fine segmentation to look for zero particles!
} 
background from fluctuations in "normal" multiparticle production. This is the motivation for our study of the multiplicity distribution at small $n$.

Quantitative results presented in this paper are based on the QCD Monte Carlo program HERWIG [4]. This program incorporates the color connections between partons, and therefore includes the natural suppression of rapidity gaps that is present in QCD, apart from the possibility of coherent colorsinglet exchange. It therefore provides a proper model for the background to rapidity gap physics. The simulation also models the production and decay of many of the known low-mass hadronic resonances. These create short-range rapidity correlations that strongly influence the multiplicity distribution in small intervals. The Monte Carlo also provides an opportunity to appraise the standard practice of substituting the easily-measured pseudo-rapidity variable $\eta=\log \cot \frac{\theta}{2}=\frac{1}{2} \log \left[\left(|\vec{p}|+p_{z}\right) /\left(|\vec{p}|-p_{z}\right)\right]$ for the more natural true rapidity $y=\frac{1}{2} \log \left[\left(E+p_{z}\right) /\left(E-p_{z}\right)\right]$.

There is no guarantee, of course, that Monte Carlo predictions for the multiplicity are correct. But it is not unreasonable to expect that formulae that will be adequate to parametrize the eventual experiments should be at least flexible enough to fit the simulated data. When real data become available, one may hope to tune the Monte Carlo parameters to improve the accuracy of the simulation.

Parametrizations based on the simulation may also be useful in correcting actual data for losses due to incomplete acceptance. This is especially important for the major detectors $\mathrm{CDF}$ and $\mathrm{D} \varnothing$, which were not designed to measure particles with transverse momenta below $\lesssim 0.2 \mathrm{GeV} /$ c. On the other hand, we will use the simulation to show in Sect. 14 that it may actually be desirable to neglect particles with very low $p_{\perp}$, since that region is overly sensitive to contamination by particles produced in the decays of resonances that are far away in rapidity, and since particles other than photons at small $|\eta|$ are kinematically suppressed there anyway.

An outline of the paper is as follows. Sect. 2 introduces some useful mathematical tools for working with multiplicity distributions - many of which have been suggested previously [12]. Sect. 3] describes results from a HERWIG simulation that is loosely applicable to experiments in progress at CDF and DØ. Sect. 4 examines the region of very low $p_{\perp}$. Sect. 5 summarizes principal conclusions. 


\section{Theoretical Tools}

\subsection{Generating Function}

Our subject is $\left\{P_{n}\right\}$, the set of probabilities to observe $n$ particles in an event in a selected region of phase space. The region is generally defined in terms of pseudo-rapidity $\eta$, or in terms of the Lego variables $\eta$ and azimuthal angle $\phi$. The particles are mainly $\pi^{ \pm}$, and $\gamma$ from $\pi^{0}$ decay, with average transverse momenta of a few hundred $\mathrm{MeV} / \mathrm{c}$.

The distribution is conveniently represented by the generating function

$$
g(x) \equiv \sum_{n=0}^{\infty} P_{n} x^{n}
$$

which carries all of the information of $\left\{P_{n}\right\}$. The factorial moments are related to the behavior of $g(x)$ in the limit $x \rightarrow 1$ :

$$
\begin{aligned}
g(1) & =1 \\
g^{\prime}(1) & =\langle n\rangle \\
g^{\prime \prime}(1) & =\langle n(n-1)\rangle \\
g^{\prime \prime \prime}(1) & =\langle n(n-1)(n-2)\rangle \\
& \ldots
\end{aligned}
$$

Meanwhile, the low multiplicity region we are interested in is contained in the behavior as $x \rightarrow 0$ :

$$
\begin{aligned}
g(0) & =P_{0} \\
g^{\prime}(0) & =P_{1} \\
g^{\prime \prime}(0) / 2 ! & =P_{2} \\
g^{\prime \prime \prime}(0) / 3 ! & =P_{3} \\
& \ldots
\end{aligned}
$$

In principle, $P_{n}$ is exactly zero beyond some large maximum $n$, because the energy in the event is finite; so $g(x)$ is a high-order polynomial. In practice, however, $P_{n}$ falls smoothly and rapidly (perhaps exponentially) at large $n$, and becomes immeasurably small long before the maximum value is approached. Hence it is appropriate to approximate $g(x)$ by an analytic 
function, whose infinite series converges at least out to $|x|=1$ in the complex plane in view of the fact that $g(1)=1$.

The analytic behavior of $g(x)$ can be useful. For if one has an analytic expression for $g$, from a model or simply a parametrization, a convenient method to calculate the corresponding probabilities is to integrate $g(x) x^{-n-1}$ numerically around the unit circle in the complex plane and use Cauchy's theorem to obtain $P_{n}$.

\subsubsection{Cluster Decay Theorem}

The generating function is a convenient tool for analyzing models in which "clusters" decay independently to make the observed hadrons. The clusters can be low-mass objects such as those assumed in QCD Monte Carlo simulations at a low $Q^{2}$ non-perturbative scale, or the hypothetical objects in branching models, or any of the large number of hadronic resonances that are the immediate ancestors of most observed hadrons.

The connection is as follows: if $P_{n}^{(1)}$ is the probability to produce $n$ clusters and $P_{n}^{(2)}$ is the probability for a cluster to decay into $n$ particles, then the overall distribution of particles $\left\{P_{n}\right\}$ is given by the generating function relation

$$
g(x)=g^{(1)}\left(g^{(2)}(x)\right)
$$

assuming that the clusters decay independently. Proof of this relation follows from the obvious expression:

$$
P_{n}=\sum_{j=0}^{\infty} P_{j}^{(1)} \sum_{n_{1}=0}^{\infty} P_{n_{1}}^{(2)} \cdots \sum_{n_{j}=0}^{\infty} P_{n_{j}}^{(2)} \delta_{n_{1}+\cdots+n_{j}, n} .
$$

The proof is easily generalized to show $g(x)=g^{(1)}\left(g^{(2)}\left(g^{(3)}(x)\right)\right)$ for independent decay of independently-decaying clusters, etc.

A simple but useful special case of this theorem applies to detection efficiencies. If $Q<1$ is the detection probability ("efficiency") for a single particle, one can think of the particle as a 'cluster' with $P_{1}=Q$ and $P_{0}=1-Q$. The effect of the inefficiency can therefore be expressed by $g[x] \rightarrow g[1-Q(1-x)]$. 


\subsubsection{Independent Sources Theorem}

The generating function is also a convenient tool for analyzing models in which the observed hadrons come from two or more statistically independent sources. An important example that we will use in Sect. 3.2 occurs in simulation programs - and perhaps also in nature - where in addition to particles resulting from a QCD hard scattering and its associated radiation, there are particles in the final state known as the "soft background event", from soft interactions between the other partons in the initial composite hadrons. This possibility of background particles leads to the notion of a survival probability for rapidity gaps [9, 13. Another example that is important for us are particles that appear far outside the cone of a jet, as a result of sequential decays of hadronic resonances produced inside the jet cone.

The relevant theorem is as follows: if two independent sources have probability distributions $\left\{P_{n}^{(1)}\right\}$ and $\left\{P_{n}^{(2)}\right\}$, then both together result in

$$
g(x)=g^{(1)}(x) \times g^{(2)}(x)
$$

Proof follows directly from

$$
P_{n}=\sum_{n_{1}=0}^{\infty} P_{n_{1}}^{(1)} \sum_{n_{2}=0}^{\infty} P_{n_{2}}^{(2)} \delta_{n_{1}+n_{2}, n} .
$$

For the lowest multiplicities, Eq. (7) takes the obvious forms

$$
\begin{aligned}
& P_{0}=P_{0}^{(1)} P_{0}^{(2)} \\
& P_{1}=P_{0}^{(1)} P_{1}^{(2)}+P_{1}^{(1)} P_{0}^{(2)} .
\end{aligned}
$$

The theorem can be generalized to

$$
\log g(x)=\log g^{(1)}(x)+\cdots+\log g^{(N)}(x)
$$

for combining $N$ independent sources. Thus on a logarithmic scale, generating functions from independent sources are additive.

\subsection{Density Function}

Intuitively, we want to make a smooth parametrization of the multiplicity distribution for $n>0$, and extrapolate it to $n=0$ to see if there is an anomalous 
contribution that would signal rapidity gap physics. The parametrization is not a trivial matter, because $P_{n}$ varies rapidly with $n$ at small $n$, especially for large rapidity intervals where $\langle n\rangle$ is large.

A representation of the probability distribution that I find to be useful describes it as a continuous superposition of Poissons:

$$
P_{n}=\int_{0}^{\infty} d z \rho(z) e^{-z} z^{n} / n !
$$

The density function $\rho(z)$ is the relative probability to have a Poisson process of average multiplicity $z$. Mathematically, $\rho(z)$ is the Laplace transform of the generating function:

$$
g(1-x)=\int_{0}^{\infty} d z \rho(z) e^{-z x}
$$

The moments of the continuous distribution $\rho(z)$ are the factorial moments: $\int_{0}^{\infty} \rho(z) d z=1, \int_{0}^{\infty} \rho(z) z d z=\langle n\rangle$, and in general

$$
\int_{0}^{\infty} \rho(z) z^{j} d z=\langle n(n-1) \cdots(n-j+1)\rangle .
$$

The independent sources theorem Eq. (6) of Sect. 2.1.2 can be expressed in terms of density functions in the form of a convolution integral

$$
\rho(z)=\int_{0}^{z} \rho^{(1)}\left(z_{1}\right) \rho^{(2)}\left(z-z_{1}\right) d z_{1} .
$$

The density function would not of course have to be positive definite; but it turns out to be so for all distributions discussed in this paper. Smoothness of $\rho(z)$ is a good way to express the physical notion that $P_{n}$ should be a smooth function of $n$, with the possible exception of structure at or near $n=0$ from the rapidity gap physics we wish to study. The behavior of $P_{n}$ at small $n$ is governed mainly by $\rho(z)$ at small $z$. In the extreme, a term $\propto \delta(z)$ would contribute to $P_{0}$ only.

A convenient way to determine $\rho(z)$ from data in an experiment or simulation is to fit the data to a parametrization whose transform is known. We will do this using a sum of terms of the form $z^{k-1} e^{-b z}$, which correspond to the NBD discussed in Sect. 2.3. From the simulation, we will find empirically that terms with $k>1$ describe most of the distribution, so $\rho(z) \rightarrow 0$ like 
a power as $z \rightarrow 0$. To allow for the possibility that $\rho(0) \neq 0$, one can also include a term of the form

$$
(1+b z) e^{-b z}
$$

which has $\rho^{\prime}(0)=0$.

\subsection{Negative Binomial Distribution}

The Negative Binomial Distribution (NBD) is a popular phenomenological form for multiplicity distributions. It is defined by

$$
P_{n}=\left(\begin{array}{c}
n+k-1 \\
k-1
\end{array}\right)\left(\frac{k}{k+\bar{n}}\right)^{k}\left(\frac{\bar{n}}{k+\bar{n}}\right)^{n}
$$

where

$$
\left(\begin{array}{c}
n+k-1 \\
k-1
\end{array}\right) \equiv \frac{k(k+1) \cdots(k+n-1)}{n !} .
$$

It can be conveniently computed with the recurrence relation

$$
\begin{aligned}
P_{0} & =(1+\bar{n} / k)^{-k} \\
P_{n+1} & =\left(\frac{n+k}{n+1}\right)\left(\frac{\bar{n}}{\bar{n}+k}\right) P_{n} .
\end{aligned}
$$

Its factorial moments are given by $\langle n\rangle=\bar{n}$ and in general

$$
\langle n(n-1) \cdots(n-j+1)\rangle=k(k+1) \cdots(k+j-1)\left(\frac{\bar{n}}{k}\right)^{j} .
$$

Its generating function is

$$
g(x)=[1+(1-x) \bar{n} / k]^{-k} .
$$

Eq. (21) implies

$$
g^{\prime \prime} g /\left(g^{\prime}\right)^{2}=1+1 / k
$$

which could be used to test whether a distribution is of the NBD form. A related test would be to see if $g / g^{\prime}$ is a linear function of $x: g(x) / g^{\prime}(x)=$ $1 / \bar{n}+(1-x) / k$. 
The density function for the NBD is

$$
\rho(z)=\frac{b^{k}}{\Gamma(k)} z^{k-1} e^{-b z}
$$

where $b=k / \bar{n}$. It has a single peak at $z=\bar{n}(1-1 / k)$ if $k>1$, or peaks at $z=0$ for $0<k<1$. As described in Sect. 2.2, a convenient way to determine $\rho(z)$ from experiment or simulation is to fit $\left\{P_{n}\right\}$ to a superposition of NBD terms and then use Eq. (23) to get $\rho(z)$.

In the limit $k \rightarrow \infty$, the NBD reduces to a Poisson distribution corresponding to uncorrelated production: $P_{n}=\left(\bar{n}^{n} / n !\right) e^{-\bar{n}}, g(x)=e^{-(1-x) \bar{n}}$, $\rho(z)=\delta(z-\bar{n})$.

The multiplicity distributions we are interested in display a single maximum with various degrees of broadness, and fall rapidly at large $n$. The two free parameters of the NBD suffice to fit the first two moments $\langle n\rangle=\bar{n}$ and $\left\langle n^{2}\right\rangle=\bar{n}^{2}(1+1 / \bar{n}+1 / k)$, and hence the NBD can provide at least a qualitative description of the probabilities $P_{n}$ where they are large. We will see, however, that a single NBD does not fit our distributions in detail.

Experimentally, the main published data on multiplicity distributions in very high energy hadron-hadron collisions are those of UA5 for charged particles in minimum bias non-diffractive events at $\sqrt{s}=900 \mathrm{GeV}$ [14. The data for rapidity intervals $\Delta \eta \leq 2$ are well described by NBD distributions. For $2 \leq \Delta \eta \leq 5$, the data are close to NBD, although the NBD fits are not statistically acceptable. However, these data come from only a few thousand events, and therefore have rather large statistical errors where $P_{n}$ is small. They also have large systematic errors at low multiplicity, where efficiencies are hard to determine. Hence the NBD form might not be magic. NBD distributions have also been seen in other data, including $e^{+} e^{-} \rightarrow$ hadrons [1, 15] and nucleus-nucleus with low statistics [16].

A systematic study of multiplicity distributions in minimum bias and/or various hard-scattering processes at the Tevatron has yet to be carried through, although preliminary results from CDF have been presented [17]. Some useful information has been obtained by E735 [18]. It would seem that D $\varnothing$ could directly extend the measurements of UA5 to $\sqrt{s}=1800 \mathrm{GeV}$, since their lack of a magnetic field simplifies the tracking of charged particles at low momentum. 


\section{Monte Carlo Simulation}

\subsection{Hard Scattering}

The QCD Monte Carlo program HERWIG 5.7 [4] was used to simulate $p \bar{p}$ scattering at the Fermilab Tevatron energy $\sqrt{s}=1800 \mathrm{GeV}$, for final states that contain two relatively high $p_{\perp}$ jets separated widely in rapidity. We will examine the multiplicity distribution in the interval between these two "trigger jets".

Specifically, we require two jets with $p_{\perp}^{(1)}, p_{\perp}^{(2)}>30 \mathrm{GeV} / \mathrm{c},-3.5<\eta_{1}<$ $-1.5,1.5<\eta_{2}<3.5$, and $\left|\eta_{2}-\eta_{1}\right|>4$. We require there to be no additional jet with $p_{\perp}>30 \mathrm{GeV} / \mathrm{c}$ elsewhere in the event. The jets are defined by a cone algorithm that I have used previously [19], with a cone radius of 0.7 in Lego. This configuration is interesting for gap physics. It is also a good one to study from an experimental standpoint, because the region between the jets, in which the multiplicity is to be measured, is in the best region of the detectors. Indeed $\mathrm{D} \varnothing$ has already published data for a rather similar configuration [20], and further data from both $\mathrm{D} \varnothing$ [21] and CDF [22] will be forthcoming.

In leading-order $\mathrm{QCD}$, the exchange of transverse momentum between the partons that produce the trigger jets is accompanied by an exchange of color. As a result, one expects lots of gluon radiation, and hence average multiplicities greater than those seen in minimum bias events, in the interval between the jets. However, if color-singlet exchanges are significant, e.g., in the form of gluon ladders, one can also expect to observe some events with rapidity gaps [8, 9, 10].

HERWIG includes all possible QCD $2 \rightarrow 2$ tree diagrams for the hard scattering. Among these diagrams, gluon exchange dominates over quark exchange because of the large rapidity separation and the gluon's higher spin. Also, the scattering partons are mainly $q$ and $\bar{q}$ because the large sub-energy

$$
\hat{s}=2 p_{\perp}^{(1)} p_{\perp}^{(2)}\left[\cosh \left(y^{(1)}-y^{(2)}\right)-\cos \left(\phi^{(1)}-\phi^{(2)}\right)\right] \cong p_{\perp}^{(1)} p_{\perp}^{(2)} e^{\left|\eta^{(1)}-\eta^{(2)}\right|}
$$

requires them to have large momentum fractions $x_{1}$ and $x_{2}$, which are suppressed more strongly for gluons by the parton distribution functions.

HERWIG is appropriate for this simulation because it correctly includes the color structure of the QCD hard scattering. It also includes the production of many of the actual low-mass hadronic resonances, which have an important 
influence on the multiplicity distribution. A final important feature is that the program contains no color-singlet exchange, or pomeron physics in any other form, so it provides a clean model for the background to rapidity gaps.

The most recent version 5.7 of HERWIG [⿴囗十 w was used, with its default parameter values except for PTMIN $=30 \mathrm{GeV} / \mathrm{c}$ to suit our desired jet $p_{\perp}$ 's, and PRSOF $=0$ which will be discussed in Sect. 3.2. It was necessary to modify the off-the-shelf program to remove unphysical behavior that otherwise appears for our rare final state, as follows. The underlying $2 \rightarrow 2$ cross section in HERWIG is evaluated for on-mass-shell partons. But the partons are actually off shell as a result of the initial state radiation branchings that are a principal feature of the program. To enforce a reasonable consistency, we reject events for which the squared four-momentum of either initial parton is larger in magnitude than

$$
Q^{2} \equiv 2 \hat{s} \hat{t} \hat{u} / \hat{s}^{2} \hat{t}^{2} \hat{u}^{2}
$$

a symmetric measure of the hardness of the scattering. [f Events in which either observed jet axis differs by more than 1.0 in rapidity from the scattered parton $($ IHEP $=7,8)$ responsible for it are also rejected. This cut removes only $10 \%$ of the events. It guarantees that the trigger jets, which are the two largest $p_{\perp}$ jets in the event, come from the underlying hard scattering, as they should to be consistent with the approximations on which the simulation is based.

The HERWIG program was modified to improve its efficiency for generating events that satisfy our cuts, with no further change in content, by replacing its uniformly random generation of the two final rapidities in the $2 \rightarrow 2$ subprocess with an appropriately peaked one. This of course required the event weighting to be handled by the user's program.

Fig. 1 shows the multiplicity distribution, based on 130,000 Monte Carlo events, for particles with $p_{\perp}>0.2 \mathrm{GeV} / \mathrm{c}$ in a rapidity interval of length 2.5 centered between the two jets in each event. The center of the interval, $\left(\eta^{(1)}+\eta^{(2)}\right) / 2$, is distributed with a mean of 0 and a standard deviation of 0.36 . The nominal jet cones lie entirely outside the interval, since the jet axes are at least $4.0=2.5+2 \times 0.75$ units apart.

The dashed curve in Fig. 1 shows an attempt to fit the distribution with a negative binomial form. Although it has the correct qualitative behavior,

\footnotetext{
${ }^{2}$ This modification is necessary to avoid unphysical behavior in HERWIG 5.7 even though a bug corrected in that version improved the situation as compared to version 5.6.
} 
the single $N B D$ does not accurately represent $\left\{P_{n}\right\}$. T The parameters of the fit shown $(\bar{n}=14.21, k=3.78)$ were chosen to match $\langle n\rangle$ and $\left\langle n^{2}\right\rangle$, but other choices don't work much better. The NBD fit is particularly poor in the region of small $n$ that is our major interest.

The solid curve in Fig. 1 is a good fit to $\left\{P_{n}\right\}$ for all $n \neq 0$. This fit has a good $\chi^{2}$, and continues to fit at larger values of $n$ (not shown), all the way out to $n \sim 60$, beyond which statistical errors become overwhelming. The fit consists of a sum of two NBD terms. However, the density function representation $\rho(z)$ in Fig. 2 (solid curve) shows that these two terms do not describe distinct peaks, but rather overlap to form a single very smooth distribution. The distribution is qualitatively similar to, but somewhat narrower than, the single NBD approximation (dashed curve).

Although the solid curve in Fig. 1 is a smooth fit to the $n \neq 0$ data, its extrapolation to $n=0$ is 0.0018 , which underestimates the actual $P_{0}=$ 0.0035 by a factor of 1.9 . This raises a warning flag for rapidity gap searches, where the signal would correspond to just such an "extra" probability for $n=0$ ! A similar but even stronger effect occurs if all particles are included instead of just those with $p_{\perp}>.2$ : the actual value is $P_{0}=0.0014$ while the extrapolation gives 0.0003. Similar behavior also occurs if the interval is defined using true rapidity $\Delta y=2.5$ in place of $\Delta \eta=2.5: P_{0}=0.0011$, fit $=0.0002 ;$ or for a larger interval such as $\Delta \eta=3.0: P_{0}=0.0011$, fit $=$ 0.0002. The effect is present but somewhat smaller if only charged particles with $p_{\perp}>0.2$ are counted: $P_{0}=0.0093$, fit $=0.0064$, or if charged particles with all $p_{\perp}$ are counted: $P_{0}=0.0052$, fit $=0.0039$. The effect remains if all hadron resonances are made stable instead of being allowed to decay: in the interval $\Delta y=2.5$ we have $P_{0}=0.0033$, fit $=0.0014$, or in the longer interval $\Delta y=3.0$ we have $P_{0}=0.0016$, fit $=0.0005$.

These results indicate that in order to establish a rapidity gap signal experimentally, the signal will have to be large compared to the background estimated by extrapolation from larger $n$, since the extrapolation can underestimate the non-pomeron contribution.

The dotted curve in Fig. 2 shows the density function for a parametrization that fits $\left\{P_{n}\right\}$ in Fig. 1 for all $n$. The parameterization contains a term

${ }^{3}$ This result is not inconsistent with a previous claim [23 that Monte Carlo simulations are NBD. That claim is based on only 2000 events, implying large statistical errors wherever $P_{n}$ is small; and even with the large statistical errors, many of the fits described are inadequate in the sense of $\chi^{2}$. 
of the form Eq. (15) that allows the extra probability for $P_{0}$ to appear as structure in $\rho(z)$ at very small $z$ with $\rho(0) \neq 0$.

\subsection{Background Event}

The colliding $p$ and $\bar{p}$ hadrons are extended objects containing many partons. Events of the type we are interested in, where two partons interact to produce jets, will generally occur only in collisions for which the impact parameter is small. There are likely to be additional soft interactions between other constituents, of the same character as those of the typical "minimum-bias" interactions that account for the fact that the inelastic interaction probability is nearly 1.0 at small impact parameter. These additional interactions lead to the production of particles known as the "soft background event", which raise $\langle n\rangle$ and decrease $P_{0}$.

It is reasonable to assume that the soft background particles are statistically independent from the particles we have considered so far. This allows us to compute the final $\left\{P_{n}\right\}$ by combining fits to its hard and soft components, using the $g(x)=g^{(1)}(x) \times g^{(2)}(x)$ theorem (Eqs. (6) (17)) or a simple Monte Carlo. This is a significant technical help to the calculation, since $P_{0}$ is so small that it would otherwise require extremely many events from the QCD simulation to determine it accurately.

The HERWIG package contains a model for the soft background event, which was turned off by setting the parameter PRSOF $=0$ to obtain the hard scattering results discussed in Sect. 3.1 above. Turning it on in every event via $\mathrm{PRSOF}=1$ leads to soft background particle distributions that are well described (at 40, 000 event statistics) by single NBD distributions, except for a sizable extra contribution to $P_{0}$. The HERWIG model for the background event is based on the UA5 data, so it is perhaps not surprising that it has an NBD form, although this result is not obvious, since the model actually assumes an NBD form for clusters rather than for final particles. I have checked that, at any rate, the model predicts charged-particle multiplicity distributions consistent with those observed by UA5. The distributions are rather broad in the sense that the NBD parameter $k$ is small. For example, for particles in the region $\Delta \eta=2.5, p_{\perp}>0.2$ corresponding to Figs. 12 , the background NBD parameters are $k=1.8$ and $\bar{n}=15.2$, with an extra contribution of 0.03 to $P_{0}$. The origin and/or validity of the "extra"

contribution, which makes $P_{0}$ larger than any other single $P_{n}$, is unclear; so 
I have tried computing the final $\left\{P_{n}\right\}$ both with it and without it.

Including the background event, with the extra contribution to $P_{0}$ "on", changes the multiplicity distribution of Fig. 1 to that shown in Fig. 3a. An expanded view showing the details at small $n$ is shown in Fig. 3b. The mean $\langle n\rangle$ has become larger, since it is equal to the sum of the means from the hard scattering and the background; while the probabilities at small $n$ have become much smaller since, e.g., to get the extreme case $n=0$ there must be no particles in the interval from the hard scattering and also none from the soft background event (Eq. (8)). The important qualitative conclusions of Sect. 3.1 remain true with the background event included, however:

- $\left\{P_{n}\right\}$ is quite similar to a single NBD form (dashed curve, based on $\bar{n}=28.88, k=4.54$ which fit $\langle n\rangle$ and $\left.\left\langle n^{2}\right\rangle\right)$; but the single NBD does not provide a fully acceptable fit, and is particularly unsuitable for describing the low multiplicities.

- $\left\{P_{n}\right\}$ can be fit very well for all but the lowest values of $n$ by a sum of two NBD terms (solid curve).

- The accurate two-NBD fit corresponds to a single smooth peak in the Poisson density function $\rho(z)$ (Fig. 4), which is slightly narrower than the single NBD approximation.

- $P_{0}$ is larger than the fit at $n=0$ by roughly a factor of 2 .

Fig. 3 is based on 1,000,000 events. This large number of events was used to obtain sufficient statistics to show the behavior at small $n$ clearly, in view of the rather small values of $P_{n}$ there. However, the inadequacy of a single NBD fit already sets in for $\gtrsim 50,000$ events.

The data in Fig. 3b can be fit by a single NBD term, with the normalization treated as a free parameter, over the small region $1 \leq n \leq 12$. This provides an alternative way to extrapolate to $n=0$. It is consistent with the result of using the two-NBD fit to the entire $n>0$ distribution.

The entire distribution in Fig. 3, including $n=0$, can be fit by a sum of two NBD terms plus a term of the form Eq. (15) that allows $\rho(0) \neq 0$. The size of this term is in fact so small that its effect would not be visible in Fig. 4. Philosophically, however, one does not expect contributions with finite probability density at average particle number zero, apart from true rapidity gap processes. 
If, instead of counting particles, one counts cells ( "towers") of size $0.1 \times 0.1$ in $(\eta, \phi)$ space that have $E_{\perp}>0.2$, as is done in calorimeter detectors, there is essentially no change in the above results, since these cells are so small that it is rare for more than one particle to enter a given cell, even at the higher values of $n$.

An attempt was made to estimate the effect on the multiplicity distribution of the geometric acceptance and detector efficiency in the current D $\varnothing$ experiment [21]. The assumed geometric acceptance was 1.0 (perfect) for $|\eta|<1.1$, with a "hole" corresponding to the edge of the central calorimeter (0.0 for $|\eta|=1.2-1.4,0.5$ for $|\eta|=1.1-1.2$ and $|\eta|=1.4-1.5)$, and a linear fall-off from 0.7 at $|\eta|=1.5$ to 0.1 at $|\eta|=3.2$. The assumed efficiency for photons rises steeply from 0 at $p=0.2 \mathrm{GeV} / \mathrm{c}$ to 0.94 by $p=1 \mathrm{GeV} / \mathrm{c}$. The assumed efficiency for charged particles rises more slowly, reaching 0.54 at $p=1 \mathrm{GeV} / \mathrm{c}$ and 0.80 at $p=3 \mathrm{GeV} / \mathrm{c}$. For intervals of length $\Delta \eta=3.0$ between the jets, with soft background particles included, the multiplicity distribution is close to a single NBD with $\bar{n}=14.0$ and $k=4.4$. Deviations from the single NBD fit begin to appear clearly when the number of events is $\gtrsim 50,000$. A sum of two NBD terms fits the distribution even for $\sim 10^{6}$ events, with very little anomalous contribution to $P_{0}=0.0014$.

\section{Edge Effects and Transverse Momentum}

Figs. $1-4$ are based on counting particles with $p_{\perp}>0.2 \mathrm{GeV} / \mathrm{c}$. One reason to require a minimum $p_{\perp}$ is to mimic typical experimental acceptance. But by using the QCD simulation to study the types and origins of particles that

contribute to the multiplicity distribution, we will see that the $p_{\perp}$ cut also provides some theoretical benefits.

Let us focus in particular on the interval of length $\Delta \eta=2.5$ centered at $\eta=0 \pm 0.36$ considered in Sect. 3.1. The composition of particles is $51 \%$ $\gamma, 39 \% \pi^{ \pm}, 6 \% K^{ \pm}, K_{L}^{0}$, and $3 \% p, \bar{p}, n, \bar{n}$. The flood of photons is caused by the fact that true rapidity is the proper measure of longitudinal phase space. The Lorentz frame-specific pseudo-rapidity $\eta$ and the true rapidity $y$ are related by

$$
\begin{aligned}
\sinh \eta & =p_{z} / p_{\perp} \\
\sinh y & =p_{z} / \sqrt{p_{\perp}^{2}+m^{2}} .
\end{aligned}
$$


These become equal for $\left|p_{\perp}\right| \gg m$, as is always the case for the massless photon; while particles whose transverse momentum is small compared to their masses are swept out to $|\eta|>|y|$. f Imposing the cut $p_{\perp}>0.2 \mathrm{GeV} / \mathrm{c}$ eliminates much of this pseudo-rapidity effect, and changes the composition to $34 \% \gamma, 52 \% \pi^{ \pm}, 9 \% K^{ \pm}, K_{L}^{0}$, and $5 \% p, \bar{p}, n, \bar{n}$.

A benefit of imposing the minimum $p_{\perp}$ cut, in addition to experimental convenience, is that it tends to eliminate particles whose pseudo-rapidities are unrepresentative of the actual underlying physics. For example, low $p_{\perp}$ photons generally come from the decay of a $\pi^{0}$ whose true rapidity differs from that of the photon by something on the order of 0.5 units. Furthermore, the $\pi^{0}$ may come from the decay of a $\rho^{ \pm}$or other low-mass resonance that is still further away; and that resonance may itself be a resonance decay product. From the standpoint of rapidity gap physics, one would like to think of $\pi, \rho$, or higher-mass resonances alike as stable. Decay effects are simply a source of "noise" in the measurement of the rapidity of produced hadrons. This noise is significant because the intervals we are looking at are not very many units long in rapidity.

The HERWIG simulation leads to the following quantitative results for the $\Delta \eta=2.5$ interval. If all particles are included, $16 \%$ come from parent particles or resonances produced outside the corresponding interval $\Delta y=2.5$ of true rapidity. This includes $5 \%$ from parents that are more than 0.5 units outside the interval. Imposing the cut $p_{\perp}>0.2 \mathrm{GeV} / \mathrm{c}$ improves the situation considerably: only $9 \%$ come from parents outside the true interval $\Delta y=2.5$, with only $1 \%$ more than 0.5 units outside it.

Rapidity gaps are traditionally defined by a total absence of particles in a particular interval of pseudo-rapidity. We should not be single-minded about this, however, since as shown above, neglecting particles with $p_{\perp}<$ $p_{\perp}^{\mathrm{MIN}} \approx 0.2 \mathrm{GeV} / \mathrm{c}$ substantially improves the connection between the pseudorapidities of the long-lived or stable particles that are measured and the true rapidities of their parent hadrons.

An additional motive for neglecting low $p_{\perp}$ particles, aside from experimental practicality, is the following: the theoretically significant variables

\footnotetext{
${ }^{4}$ Attempts [24, 25] to measure the average charged multiplicity $d N_{\mathrm{ch}} / d \eta$ apparently have ignored this pseudo-rapidity phenomenon when extrapolating to account for the unmeasured portion of the spectrum at small $p_{\perp}$. They also use a phenomenological form $d N / d \eta d p_{\perp}^{2} \propto\left(p_{\perp}+p_{0}\right)^{-a}$ which has incorrect analytic behavior in $p_{\perp}^{2}: d N / d y d p_{\perp}^{2} \propto$ $\left(p_{\perp}^{2}+p_{0}^{2}\right)^{-a}$ would be preferable.
} 
in rapidity gap physics are generally the invariant masses of the hadronic systems to the left and right of the gap. These masses depend on the "+"

and "-" components of light-cone momentum, $\sqrt{p_{\perp}^{2}+m^{2}} e^{ \pm y}$, for which particles with very low $p_{\perp}$ are less important.

\section{Conclusion}

The ideal way to look for rapidity gaps would be to measure the multiplicity zero component $P_{0}$ as a function of interval size $\Delta \eta$. The background from non-gap physics should decline rapidly with increasing $\Delta \eta$-for example, $P_{0} \propto e^{-2 \Delta \eta}$ roughly describes the results of our simulation in the range $\Delta \eta \approx 1-3$. Any residual constant or slowly varying component at large $\Delta \eta$ is the rapidity gap signal.

Because current experiments are limited in interval size, and because it is necessary to make experimental corrections based on measurements at nonzero $n$, we have studied instead the form of the $P_{n}$ distribution in fixed regions of $\Delta \eta$. One can hope that for $\Delta \eta \simeq 3$, the rapidity gap signal will appear as an anomalously large contribution to $P_{0}$, when compared to a smooth parameterization that describes the rest of the distribution.

With the help of the QCD simulation program HERWIG, we have found suitable ways to parametrize the $P_{n}$ distribution. A particularly convenient choice is a sum of two NBD terms, which gives a very good fit, and automatically provides a simple parametrization of the generating function $g(x)$ and the Poisson density function $\rho(z)$. The smoothness of the parametrization is demonstrated by the smooth single-peak form of $\rho(z)$ (Figs. 2, 4). The absence of an anomalous contribution (or a gap signal) at very small $n$ can be characterized by a power-law behavior $\rho(z) \sim$ const $\times z^{a}$ where $a>0$ so that $\rho(0)=0$. Meanwhile, the often-used single NBD form has this property, and describes the distribution qualitatively (Fig. 3a); but is not good enough at small $n$ (Fig. 3b) for measuring the background to a rapidity gap signal unless the region of $n$ included in the fit is sharply restricted.

We find from the simulation that fits to $P_{n}$ for $n \geq 2$ can underestimate $P_{0}$ by a factor $\sim 2$. This is a cautionary tale for rapidity gap studies, because the excess $P_{0}$ has the same form as a rapidity gap signal. It will nevertheless be possible to measure true gap effects in intervals as small as $2-3$ units, provided the $n=0$ cross section turns out to be large compared to the 
extrapolation from larger $n$. This will happen if the signal turns out to be on the order of $1 \%$ or larger. Results from the experiments are eagerly awaited!

Further work is needed to create a phenomenological model to describe the rapidity gap physics itself, which contributes not only to $n=0$, but also to other low multiplicities since the gap in a given event can be just slightly shorter than the rapidity interval under consideration, and since there are edge effects associated with resonance decays as discussed in Sect. t. $^{-}$

\section{Acknowledgments}

I wish to thank J. D. Bjorken for discussions of rapidity gap physics, and H. Weerts, A. Brandt and S. Kuhlmann for discussions of the experimental situation. This work was supported in part by the Texas National Laboratory Research Commission grant to the CTEQ collaboration.

\section{References}

[1] L. Van Hove and A. Giovannini, Acta Phys. Polon. B19, 931 (1988); A. Giovannini and L. Van Hove, Acta Phys. Polon. B19, 495 (1988); R. Ugoccioni and A. Giovannini, Z. Phys. C53, 239 (1992).

[2] Wolfgang Ochs, Acta Phys. Polon. B22, 203 (1991); A. Białas and R. Peschanski, Phys. Lett. 207B, 59 (1988); J-C Pan and R.C. Hwa, Phys. Rev. D48, 168 (1993); R. Ugoccioni, et al., "Clan properties in parton showers", <hep-ph/9404202 > (Mar 1994); S.G. Matinian and E.B. Prokhorenko, Phys. Rev. D48, 5127 (1993).

[3] Yu. L. Dokshitzer, Phys. Lett. B305, 295 (1993); I. M. Dremin, Phys. Lett. B313, 209 (1993) and review article $<$ hep-ph/9406231 >

[4] G. Marchesini and B.R. Webber, Nucl. Phys. B310, 461 (1988); G. Abbiendi, I.G. Knowles, G. Marchesini, B.R. Webber, M.H. Seymour and L. Stanco, Comp. Phys. Comm. 67, 465 (1992).

[5] S. Hegyi and S. Kraznovszky, Phys. Lett. B241, 605 (1990). 
[6] J. Bachler, et al., Z. Phys. C61, 551 (1994); A. Białas and R. Peschanski, Nucl. Phys. B308, 857 (1988); L. Van Hove, Phys. Lett. B232, 509 (1989); J.M. Alberty and A. Biał, Z. Phys. C50, 315 (1991); A. Białas and M. Gadzicki, Phys. Lett. B252, 483 (1990); J-C Pan and R.C. Hwa, Phys. Rev. D46, 4890 (1992); P. Carruthers and I. Sarcević, Phys. Rev. Lett. 63, 1562 (1989), Erratum 63, 2612 (1989).

[7] A. Białas and K. Zalewski, Phys. Lett. B228, 155 (1989).

[8] A.H. Mueller and W.K. Tang, Phys. Lett. B284, 123 (1992).

[9] J.D. Bjorken, Int. J. Mod. Phys. A7, 4189 (1992); Phys. Rev. D47, 101 (1992); Phys. Rev. D45, 4077 (1992); J.D. Bjorken, et al., Phys. Lett. B286, 153 (1992).

[10] V. Del Duca and W-K Tang, Phys. Lett. B312, 225 (1993); H-J Lu and J. Milana, Phys. Lett. B313, 234 (1993); V. Del Duca and C.R. Schmidt, $<$ hep-ph 9311290 $>$; V. Del Duca, $<$ hep-ph 9308206 $>$.

[11] ZEUS Collaboration (M. Derrick et al.), Phys. Lett. B315, 481 (1993); and "Observation of jet production in deep inelastic scattering with a large rapidity gap at HERA", DESY preprint 94-063.

[12] P. Carruthers and C.C. Shih, Int. J. Mod. Phys. A2, 1447 (1987).

[13] E. Levin, Phys. Rev. D48, 2097 (1993); E. Gotsman, E. Levin and U. Maor, Phys. Lett. B309, 199 (1993).

[14] UA5 collaboration (R. Ansorge et al.), Zeit. Phys. C43, 357 (1989).

[15] A. Chaudhuri, Phys. Rev. D45, 4057 (1992) and references contained.

[16] P.L. Jain et al., Phys. Lett. B294, 27 (1992).

[17] F. Rimondi, "Multiplicity distributions in anti-p p interactions at $\mathrm{s}^{* *}(1 / 2)=1800-\mathrm{GeV}^{\prime}$, FERMILAB-CONF-93-359-E (Nov 1993).

[18] E735 Collaboration (T. Alexopoulos et al.), Phys. Rev. D48, 984 (1993);

A. Bouzas et al., Zeit. Phys. C56, 107 (1992).

[19] J. Pumplin, Phys. Rev. D44, 2025 (1991). 
[20] DØ Collaboration (S. Abachi et al.), Phys. Rev. Lett. 72, 2332 (1994).

[21] A. Brandt, private communication.

[22] S. Kuhlmann, private communication.

[23] L. Van Hove and A. Giovannini, Acta Phys. Polon. B19, 917 (1988).

[24] CDF Collaboration (F. Abe, et al.), Phys. Rev. D41, 2330 (1990).

[25] UA1-MIMI Collaboration (G. Bocquet, et al.), CERN-PPE-94-47, March 1994. 


\section{Figure Captions}

1. Multiplicity distribution for particles with $p_{\perp}>0.2$ in a pseudo-rapidity interval $\Delta \eta=2.5$ centered between jets, with "soft background event" turned off. Solid curve: good fit for $n \geq 1$; dashed curve: single NBD fit.

2. Poisson density function representations $\rho(z)$ of the fits shown in Fig. 1. Dotted curve: density function of fit to all data, including $n=0$, in Fig. 1.

3. (a) Multiplicity distribution similar to Fig. 1, with "soft background event" included. (b) Expanded view of the distribution at small $n$. Solid curve: good fit for $n \geq 2$; dashed curve: single NBD fit.

4. Poisson density function representations of the fits in Fig. 3. 
This figure "fig1-1.png" is available in "png" format from: http://arxiv.org/ps/hep-ph/9407332v1 
This figure "fig1-2.png" is available in "png" format from: http://arxiv.org/ps/hep-ph/9407332v1 
This figure "fig1-3.png" is available in "png" format from: http://arxiv.org/ps/hep-ph/9407332v1 
This figure "fig1-4.png" is available in "png" format from: http://arxiv.org/ps/hep-ph/9407332v1 
This figure "fig1-5.png" is available in "png" format from: http://arxiv.org/ps/hep-ph/9407332v1 